Published version available at: https://doi.org/10.1016/j.combustflame.2020.08.050

\title{
Superadiabatic small-scale combustor with counter-flow heat exchange: flame structure and limits to narrow-channel approximation
}

\author{
Vadim N. Kurdyumov ${ }^{1}$, Daniel Fernández-Galisteo, \\ Carmen Jiménez \\ Department of Energy, CIEMAT, Avda. Complutense 40, 28040 Madrid, Spain
}

\begin{abstract}
Small-scale superadiabatic combustors with counter-flow heat exchange segments of finite length are studied using irreversible Arrhenius kinetics. First, the complete twodimensional conservation equations for the gas phase and the solid walls separating the channels are investigated numerically. Next, a simplified one-dimensional model, also known as the narrow-channel approximation, is derived asymptotically and the results are compared with those of the complete model. The main aim of the study is to assess the applicability limits of the simplified model. The analysis shows that this approximation provides valid results beyond its strict limits of validity.
\end{abstract}

\section{Introduction}

Recent challenges for combustion science linked to the reduction of harmful emissions and the increase of efficiency require the development and validation of new combustion applications that use low-energy containing mixtures. Among them we find small-scale devices designed for hydrogen production and based on reforming rich fuel-air mixtures by means of partial oxidation. Another class of such devices consists in small burners for low-energy fuel-residual gases to provide, in particular, energy supply for portable electronic devices. In both cases, the direct chemical conversion per se is hardly viable due to low heat output and high heat losses. Comprehensive reviews regarding these micro combustion devices can be found in [1-6].

A viable solution for combustion of low-energy-containing mixtures might be the use of small-scale devices with heat exchanging between adjacent counter-flow streams. The idea of

\footnotetext{
${ }^{1}$ Corresponding author
} 
this sort of devices should be traced back to [7-9] and the corresponding studies have received significant attention over the past two decades [10-19].

An increase in the efficiency of heat exchange between counterflow streams can be achieved by reducing the thickness of the channels when it becomes comparable with the thermal thickness of the flame. A simple one-dimensional model for such devices was developed in [11-13] where the flame-sheet approximation for the reaction rate was also adopted. These simplifications allowed an analytical treatment both for steady state solutions and for their stability analysis. Various numerical studies based on two-dimensional governing equations were carried out in [14-17] for different burner configurations. The influence of power extraction on combustion regimes has been reported recently in [18] and the production viability of hydrogen-rich syngas from methanol has been explored in [19].

The description of processes in such devices requires a significant number of parameters, to account for the physicochemical properties of combustible mixtures and for the geometrical characteristics of the devices themselves. For this reason, the use of simplified one-dimensional models for the parametric analysis has a clear advantage due to the lower computational cost of numerical simulations. Typically, these one-dimensional models are built in an ad-hoc manner, without proper and rigorous studies of their applicability. In contrast to this, we believe that proper construction of such simplified models should be based on asymptotic methods. It is also well known that asymptotic considerations provide approximations that could very well be valid beyond their strict (mathematic) limit of validity. However, the range of validity cannot be obtained within the framework of the asymptotic methods themselves. Instead, it has to be deduced from the comparison of results of the one-dimensional model to results of the complete multidimensional system.

The purpose of the current work is to justify the use of one-dimensional models for devices with heat recirculation and to determine the limits of their applicability. With this aim, the two-dimensional model taken as a starting point is investigated numerically first. After that, the limit of narrow channels is developed and the results are compared with those obtained for the complete problem. The article is arranged as follows: in Section 2, the general formulation is given; a short description of the numerical treatment is given in Section 3; the asymptotic considerations leading to the one-dimensional narrow channel approximation are presented in Section 4; the asymptotic results are compared with the direct two-dimensional numerical calculations in Section 5; in addition, the high activation energy limit is used within the narrow channel approximation in Section 6. Finally, conclusions are drawn in the last section. 


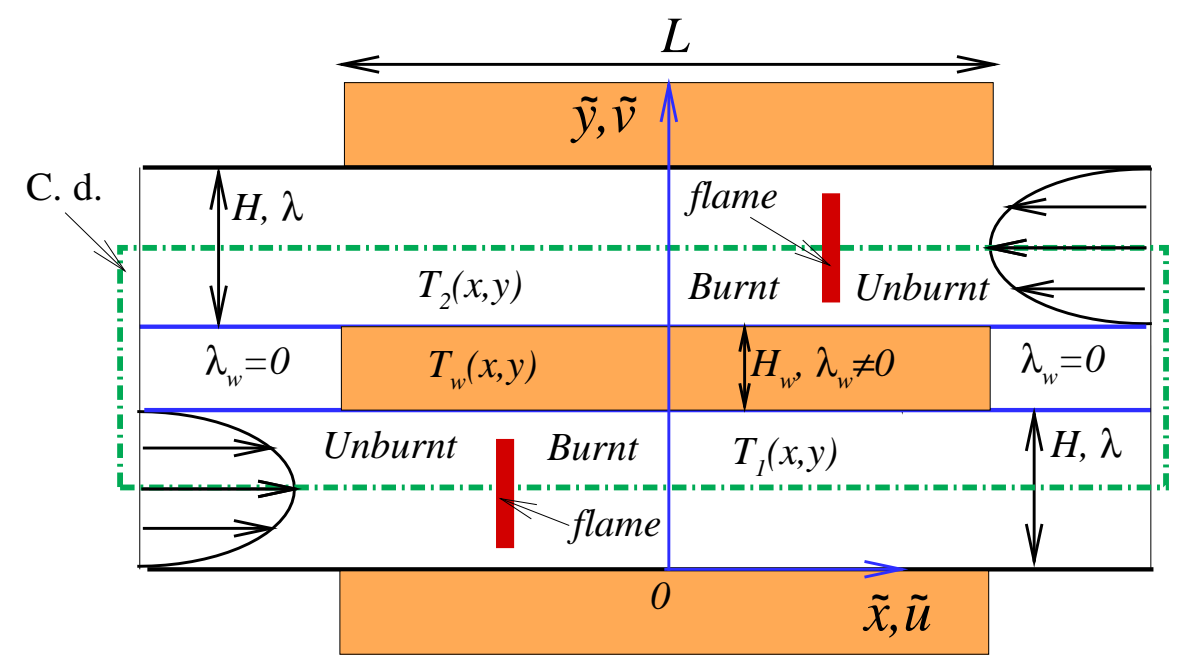

Figure 1: Sketch of the problem, coordinate system and opposite-velocity profiles in two channels; the computational domain is marked with a dash-dot rectangle.

\section{General formulation}

A schematic representation of the idealized counter-flow combustor under consideration is given in Fig. 1. The device consists of an array of planar parallel channels of height $H$ separated from each other by walls of thickness $H_{w}$. A combustible mixture at initial temperature $T_{0}$ flows in opposite directions in adjacent channels with a fixed mass flow rate, $M_{0}$, in every channel. The configuration is periodic in the $y$-direction. We assume that the walls are thermally adiabatic except for segments of length $L$ where heat exchange between the opposing streams takes place. The origin of a coordinate system, $\tilde{x}=0$, is fixed in the middle of the conducting segments, as shown in Fig. 1. Here and below " $\sim$ is used to denote dimensional variables if the same notations are used for dimensional and dimensionless variables. In what follows, cases with $h_{w}=H_{w} / H \sim O(1)$ and $L \gg H$ are considered.

In the present study all flames are symmetric with respect to the corresponding channel midplanes. Periodicity of the system allows to reduce the domain to that marked in Fig. 1 with a dash-dot line. The possible impact of non-symmetric flames similar to those reported in [20-23] is out of the scope of the present study and will be reported elsewhere.

In what follows, subindexes 1 and 2 are used for channels with rightward and leftward flow directions, respectively, and subindex $w$ for the wall, as sketched in Fig. 1. The subindexes are 
omitted in general formulae (e.g. the governing equations) applicable to any channel.

When the mixture is ignited and the flames are established in the channels, their structure and location depend on the flow rate and the heat recirculation that takes place between the cold fresh mixture and hot products. The chemical reaction in the gas phase is modeled by a global irreversible step of the form Fuel + Oxidizer $\rightarrow$ Products. It is assumed that the mixture has a deficient component (fuel or oxidizer) and changes in the non-deficient mass fraction remain small. The mass of deficient reactant consumed per unit volume and unit time is $\tilde{\omega} \sim \tilde{\rho}^{2} Y_{F} Y_{O}$, where $Y_{F}, Y_{O}$ are the mass fractions of the fuel and oxidizer, and $\tilde{\rho}$ is the density of the mixture. Then, assuming that the reaction is first order with respect to the mass fraction of the deficient reactant, $Y$, the reaction rate takes the form

$$
\tilde{\omega}=\mathcal{B} \tilde{\rho}^{2} Y e^{-E / \mathcal{R} T}
$$

where $E$ is the overall activation energy, $T$ is the temperature, $\mathcal{R}$ is the gas constant, and $\mathcal{B}$ is the appropriately defined pre-exponential factor. For example, $\tilde{\omega}$ stands for the oxidizer consumption rate in rich mixtures.

When the combustion kinetics is modeled by Eq. (1) a planar flame solution characterized by a planar, isobaric, adiabatic flame speed, $S_{L}$, exists always, in contrast to more complex chemical models where, for example, a flammability limit exists. The non-dimensionalization procedure is based on $S_{L}$, the thermal flame thickness defined as $\delta_{T}=\lambda / \rho_{0} c_{p} S_{L}$ (representing the thickness of a planar adiabatic and isobaric flame) and the adiabatic flame temperature $T_{a}=$ $T_{0}+Q Y_{0} / c_{p}$. Here $\lambda$ and $c_{p}$ (assumed constant) are the thermal conductivity and the specific heat capacity (at constant pressure) of the mixture, respectively, $Q$ is the total heat of combustion per unit mass of deficient component and $Y_{0}$ is the initial deficient mass fraction. The parameters $a=H / \delta_{T}$ and $\ell=L / \delta_{T}$ stand for the channel width and the length of the heat exchange segments, in units of the flame thickness $\delta_{T}$.

The states of unburned mixture, $\rho_{0}, p_{0}$ and $T_{a}-T_{0}=Q Y_{0} / c_{p}$, are used as scaling factors for the density $\rho$, pressure $p$ and temperature $T$. The upstream mean flow velocity $U_{0}=M_{0} / \rho_{0}$ controls the flow rate through the system. In order to investigate the asymptotic limit of narrow channels, $a \ll 1$, different length and velocity scales are convenient along the axial and transverse directions. The thermal flame thickness, $\delta_{T}$, and the mean velocity, $U_{0}$, are used for the $x$-variables. The values $H$ and $\left(H / \delta_{T}\right) U_{0}$ are used for the corresponding $y$-variables. We write

$$
\begin{gathered}
x=\tilde{x} / \delta_{T}, \quad y=\tilde{y} / H, \quad t=S_{L} \tilde{t} / \delta_{T}, \quad u=\tilde{u} / U_{0}, \quad v=\tilde{v} / a U_{0}, \quad \rho=\tilde{\rho} / \rho_{0}, \\
p=a^{2}\left(\tilde{p}-p_{0}\right) /\left(\rho_{0} S_{L} U_{0}\right), \quad \theta=\left(T-T_{0}\right) /\left(T_{a}-T_{0}\right), \quad \theta_{w}=\left(T_{w}-T_{0}\right) /\left(T_{a}-T_{0}\right) .
\end{gathered}
$$


Assuming constant transport coefficients and a low-Mach number approximation, the standard governing equations take the following (dimensionless) form: the gas phase:

$$
\begin{gathered}
\frac{\partial \rho}{\partial t}+\frac{\partial(m \rho u)}{\partial x}+\frac{\partial(m \rho v)}{\partial y}=0 \\
\rho\left(\frac{\partial u}{\partial t}+m u \frac{\partial u}{\partial x}+m v \frac{\partial u}{\partial y}\right)=-\frac{1}{a^{2}} \frac{\partial p}{\partial x}+\operatorname{Pr}\left[\frac{1}{a^{2}} \frac{\partial^{2} u}{\partial y^{2}}+\frac{4}{3} \frac{\partial^{2} u}{\partial x^{2}}+\frac{1}{3} \frac{\partial^{2} v}{\partial x \partial y}\right] \\
\rho\left(\frac{\partial v}{\partial t}+m u \frac{\partial v}{\partial x}+m v \frac{\partial v}{\partial y}\right)=-\frac{1}{a^{4}} \frac{\partial p}{\partial y}+\operatorname{Pr}\left[\frac{1}{a^{2}}\left(\frac{4}{3} \frac{\partial^{2} v}{\partial y^{2}}+\frac{1}{3} \frac{\partial^{2} u}{\partial x \partial y}\right)+\frac{\partial^{2} v}{\partial x^{2}}\right] \\
\rho\left(\frac{\partial \theta}{\partial t}+m u \frac{\partial \theta}{\partial x}+m v \frac{\partial \theta}{\partial y}\right)=\frac{\partial^{2} \theta}{\partial x^{2}}+\frac{1}{a^{2}} \frac{\partial^{2} \theta}{\partial y^{2}}+\omega \\
\rho\left(\frac{\partial Y}{\partial t}+m u \frac{\partial Y}{\partial x}+m v \frac{\partial Y}{\partial y}\right)=\frac{1}{L e}\left(\frac{\partial^{2} Y}{\partial x^{2}}+\frac{1}{a^{2}} \frac{\partial^{2} Y}{\partial y^{2}}\right)-\omega
\end{gathered}
$$

the solid phase:

$$
\frac{\partial \theta_{w}}{\partial t}=\alpha\left(\frac{\partial^{2} \theta_{w}}{\partial x^{2}}+\frac{1}{a^{2}} \frac{\partial^{2} \theta_{w}}{\partial y^{2}}\right)
$$

the ideal-gas state equation:

$$
\rho(1+q \theta)=1
$$

Notice that the dimensionless flow rate $m=U_{0} / S_{L}=M_{0} / \rho_{0} S_{L}$ appears explicitly as a factor in Eqs. (3)-(7). In the above equations $q=Q Y_{0} / c_{p} T_{0}=\left(T_{a}-T_{0}\right) / T_{0}$ represents the heat release parameter, $\operatorname{Pr}=\mu c_{p} / \lambda$ is the Prandtl number, with $\mu$ the viscosity of the mixture, $L e=\lambda / \rho_{0} c_{p} \mathcal{D}$ is the Lewis number, with $\mathcal{D}$ the diffusivity of the deficient reactant and $\alpha=$ $\left(\lambda_{w} / \lambda\right) \cdot\left[\left(\rho_{0} c_{p}\right) /\left(\rho_{w} c_{w}\right)\right]$ represents the dimensionless wall thermal diffusivity, with $\rho_{w}$ and $c_{w}$ the density and the heat capacity of the wall material. One can see that for steady cases, namely when $\partial / \partial t \equiv 0$, the parameter $\alpha$ has no effect on the solutions.

The dimensionless reaction rate takes the form

$$
\omega=\frac{\beta^{2}}{2 L e u_{p}^{2}}(1+q)^{2} \rho^{2} Y \exp \left\{\frac{\beta(\theta-1)}{1+q(\theta-1) /(1+q)}\right\},
$$

where $\beta=E\left(T_{a}-T_{0}\right) / \mathcal{R} T_{a}^{2}$ is the Zel'dovich number. The factor $u_{p}=S_{L} / U_{L}$ appearing in Eq. (10) has been introduced to account for the difference between the asymptotic value of the laminar flame speed,

$$
U_{L}=\sqrt{\frac{2\left(\lambda / c_{p}\right) \mathcal{B} L e}{\beta^{2}}} \frac{T_{0}}{T_{a}} e^{-E / 2 \mathcal{R} T_{a}}
$$


obtained for large activation energy $(\beta \rightarrow \infty)$ and the planar isobaric laminar flame speed, $S_{L}$, calculated for a finite $\beta$, see [24]. Computed values of $u_{p}$ for $\beta=10$ and selected values of $q$ and $L e$ are given in [25]. The factor $u_{p}$ ensures that the dimensionless planar flame velocity is equal to one for finite values of $\beta$.

Equations (3)-(9) are to be solved subject to the following boundary conditions:

- along the solid walls, at $y=1$ and $y=1+h_{w}$,

$$
u=v=\frac{\partial Y}{\partial y}=0, \quad \theta=\theta_{w}, \quad \frac{\partial \theta}{\partial y}= \begin{cases}\frac{1}{2} K a^{2} h_{w} \frac{\partial \theta_{w}}{\partial y}, & |x|<\ell / 2, \\ 0, & |x|>\ell / 2\end{cases}
$$

- along the midplane of the channels, at $y=1 / 2$ and $y=3 / 2+h_{w}$,

$$
\frac{\partial u}{\partial y}=v=\frac{\partial \theta}{\partial y}=\frac{\partial Y}{\partial y}=0
$$

- along the lateral conducting wall segments, at $1<y<1+h_{w}$ and $x= \pm \ell / 2$,

$$
\frac{\partial \theta_{w}}{\partial x}=0
$$

- far upstream the channels,

$$
u \rightarrow \pm 6 y(1-y), \quad v \rightarrow 0, \quad \theta \rightarrow 0, \quad Y \rightarrow 1
$$

- at the outlet of the channels,

$$
\frac{\partial u}{\partial x}=\frac{\partial \theta}{\partial x}=\frac{\partial Y}{\partial x}=0 .
$$

The Poiseuille velocity profile is stated in Eq. (14) far upstream in the fresh mixture with sign " + " and " - " for the channels with rightward and leftward flows, respectively.

Notice that the heat exchange parameter

$$
K=\frac{2 \lambda_{w}}{\lambda a^{2} h_{w}}=2 \frac{\lambda_{w}}{\lambda} \cdot \frac{\delta_{T}^{2}}{H H_{w}}
$$

has been introduced in the boundary conditions (11) instead of using of the parameter $\lambda_{w} / \lambda$ directly. It will be shown in Section 4 that this value is preferable in the cases of narrow channels, as $a \rightarrow 0$. Moreover, the results presented below also show that this scaling is also useful even for $a=O(1)$. From the other side the use of $K$ for wide channels, when $a \gg 1$, becomes meaningless. 
In the following, the flame position $x_{f}$ is defined as the point along the channel midplane at which the reaction rate $\omega$ reaches its maximum value. We will use also the maximum temperature (which coincides with the flame temperature) attained along the midplane in order to characterize the flame. In the majority of the cases presented below the temperature and mass fraction distributions in channels 1 and 2 resulted symmetric with respect to $x=0$. This means that $\theta_{1}\left(x, y_{1}\right)=\theta_{2}\left(-x, y_{2}\right)$, where the coordinates $y_{1}$ and $y_{2}$ are symmetric with respect to the wall midplane $y_{*}=1+h_{w} / 2$, namely $y_{2}=2+h_{w}-y_{1}$. Evidently, the maximum temperatures in both channels are equal and the flame positions satisfy $x_{f 1}=-x_{f 2}$.

The numerical calculations were carried out in the computational domain marked with a dashed-dot line rectangle in Fig. 1, that is, in the two channels. Nevertheless, when the solutions are symmetric, the distribution of variables corresponding to the rightward flow channel will be sufficient to characterize the process. All the calculations reported below were carried out for $L e=1, \operatorname{Pr}=0.7$ and $q=5$. The value of the Zel'dovich number was fixed at $\beta=10$ in the majority of cases unless otherwise specified.

\section{Numerical treatment}

All two-dimensional computations were carried out in a finite domain, $-x_{\max }<x<x_{\max }$, with $x_{\max }$ significantly larger than $\ell / 2$. The spatial derivatives were discretized on a uniform grid using second order, three-point central differences. The steady counterpart $(\partial / \partial t=0)$ of the governing equations was solved using a Gauss-Seidel method with over-relaxation. The solutions were obtained using two iterating methods. In the first method, the value of the flow rate, $m$, was fixed. Only solutions belonging to the stable branch (see below) can be calculated using this method. In the second method, the temperature was fixed at a point with $x=x_{*}$ (and $y=y_{*}$, for two-dimensional calculations) in the rightward flow channel, imposing there $\theta_{1}=\theta_{*}$ while the value of $m$ was calculated iteratively (also with a Gauss-Seidel procedure). The numerical values of $\theta_{*}$ used in the calculations were $\theta_{*}=0.5 \div 0.7$.

The steady-state solutions of Eqs. (3)-(8) were calculated using a standard method introducing the stream function $\psi$ and the vorticity $\zeta$. With $\psi$ defined from $\rho u=\partial \psi / \partial y, \rho v=-\partial \psi / \partial x$, and the vorticity as $\zeta=a^{2} \partial v / \partial x-\partial u / \partial y$, the Navier-Stokes equations are reduced to

$$
m \rho u \frac{\partial \zeta}{\partial x}+m \rho v \frac{\partial \zeta}{\partial y}=\operatorname{Pr}\left(\frac{\partial^{2} \zeta}{\partial x^{2}}+\frac{1}{a^{2}} \frac{\partial \zeta}{\partial y^{2}}\right)+J
$$




$$
a^{2} \frac{\partial}{\partial x}\left(\frac{1}{\rho} \frac{\partial \psi}{\partial x}\right)+\frac{\partial}{\partial y}\left(\frac{1}{\rho} \frac{\partial \psi}{\partial y}\right)=-\zeta
$$

where

$$
J=m\left[\frac{\partial(\rho u)}{\partial y} \frac{\partial u}{\partial x}-a^{2} \frac{\partial(\rho u)}{\partial x} \frac{\partial v}{\partial x}+\frac{\partial(\rho v)}{\partial y} \frac{\partial u}{\partial y}-a^{2} \frac{\partial(\rho v)}{\partial x} \frac{\partial v}{\partial y}\right]
$$

denotes the vorticity production.

\section{Narrow-channel approximation}

A convincing asymptotic reduction of the two-dimensional problem to its one-dimensional counterpart has evident numerical advantages for narrow channels. In addition to reducing numerical costs, this allows to recover properly the heat-exchange parameter which should be used at low $a$.

The asymptotic procedure carried out below is similar to that described in [25] where a single adiabatic channel was considered. It will not be reproduced here in all details. For the sake of brevity we reproduce only the temperature equations while for the rest of variables the equivalent asymptotic reduction can be easily deduced.

Assuming (formally) that all parameters are fixed and $a \ll 1$, we expand all independent variables in power series of $a^{2}$, i.e. in the form $f=f^{(0)}+a^{2} f^{(1)}+\ldots$, for a generic variable $f$. To the leading order, Eqs. (6) and (8) are reduced to

$$
\frac{\partial^{2} \theta_{1}^{(0)}}{\partial y^{2}}=\frac{\partial^{2} \theta_{2}^{(0)}}{\partial y^{2}}=\frac{\partial^{2} \theta_{w}^{(0)}}{\partial y^{2}}=0
$$

These equations imply linear with $y$ temperature profiles inside the channels and the wall, as a first approximation. Applying Eq. (12) leads to

$$
\theta_{1,2}^{(0)}=\theta_{1,2}^{(0)}(x, t) \quad \text { and } \quad \theta_{w}^{(0)}=\theta_{1}^{(0)}+\frac{\theta_{2}^{(0)}-\theta_{1}^{(0)}}{h_{w}}(y-1) .
$$

Similarly $Y_{1,2}^{(0)}=Y_{1,2}^{(0)}(x, t)$, and, consequently, $\rho_{1,2}^{(0)}$ and $\omega_{1,2}^{(0)}$ are all functions of $x$ and $t$ only.

We now proceed to the next order in $a^{2}$. Equation (6) written for the first and second channels gives

$$
\begin{aligned}
& \frac{\partial^{2} \theta_{1}^{(1)}}{\partial y^{2}}=\rho_{1}^{(0)} \frac{\partial \theta_{1}^{(0)}}{\partial t}+m \rho_{1}^{(0)} u_{1}^{(0)} \frac{\partial \theta_{1}^{(0)}}{\partial x}-\frac{\partial^{2} \theta_{1}^{(0)}}{\partial x^{2}}-\omega_{1}^{(0)} \\
& \frac{\partial^{2} \theta_{2}^{(1)}}{\partial y^{2}}=\rho_{2}^{(0)} \frac{\partial \theta_{2}^{(0)}}{\partial t}+m \rho_{2}^{(0)} u_{2}^{(0)} \frac{\partial \theta_{2}^{(0)}}{\partial x}-\frac{\partial^{2} \theta_{2}^{(0)}}{\partial x^{2}}-\omega_{2}^{(0)} .
\end{aligned}
$$


The boundary conditions (11) for the temperature at $y=1$ and $y=1+h_{w}$ are reduced to

$$
\frac{\partial \theta_{1,2}^{(1)}}{\partial y}= \begin{cases}\frac{1}{2} K h_{w} \frac{\partial \theta_{w}^{(0)}}{\partial y}=\frac{1}{2} K\left(\theta_{2}^{(0)}-\theta_{1}^{(0)}\right), & |x|<\ell / 2 \\ 0, & |x|>\ell / 2 .\end{cases}
$$

Integrating Eqs. (19) and (20) from $y=1 / 2$ to $y=1$ and from $y=1+h_{w}$ to $y=3 / 2+h_{w}$, respectively, and using Eq. (21) yields

$$
\begin{aligned}
& \rho_{1} \frac{\partial \theta_{1}}{\partial t}+m \rho_{1} U_{1} \frac{\partial \theta_{1}}{\partial x}=\frac{\partial^{2} \theta_{1}}{\partial x^{2}}+\omega_{1}-K \mathcal{H}\left(\theta_{1}, \theta_{2}\right) \\
& \rho_{2} \frac{\partial \theta_{2}}{\partial t}+m \rho_{2} U_{2} \frac{\partial \theta_{2}}{\partial x}=\frac{\partial^{2} \theta_{2}}{\partial x^{2}}+\omega_{2}+K \mathcal{H}\left(\theta_{1}, \theta_{2}\right)
\end{aligned}
$$

where the upper index has been dropped for simplicity. Here the term

$$
K \mathcal{H}\left(\theta_{1}, \theta_{2}\right)=\left\{\begin{array}{cl}
K\left(\theta_{1}-\theta_{2}\right), & |x|<\ell / 2 \\
0, & |x|>\ell / 2
\end{array}\right.
$$

describes the heat-exchange with $K$ given by Eq. (16). $U_{1}=\int_{0}^{1} u_{1}^{(0)} d y$ and $U_{2}=\int_{1+h_{w}}^{2+h_{w}} u_{2}^{(0)} d y$ denote the mean flow velocities. The continuity and mass fraction equations are reduced to

$$
\begin{gathered}
\frac{\partial \rho_{i}}{\partial t}+\frac{\partial\left(m \rho_{i} U_{i}\right)}{\partial x}=0 \\
\rho_{i} \frac{\partial Y_{i}}{\partial t}+m \rho_{i} U_{i} \frac{\partial Y_{i}}{\partial x}=\frac{1}{L e} \frac{\partial^{2} Y_{i}}{\partial x^{2}}-\omega_{i}
\end{gathered}
$$

where $i=1,2$ and $\rho_{i}$ and $\omega_{i}$ are given by Eqs. (9) and (10), respectively.

Summing up the temperature equation multiplied by $q$ and the continuity equation multiplied by $(1+q \theta)$ and using the equation of state (9) gives

$$
m \frac{\partial U_{i}}{\partial x}=q\left[\frac{\partial^{2} \theta_{i}}{\partial x^{2}}+\omega_{i} \mp K \mathcal{H}\left(\theta_{1}, \theta_{2}\right)\right]
$$

This equation can be used instead of Eq.(25) to close the formulation.

As showed in [25], the mean velocities are related to the corresponding pressure gradients in every channel through Darcy's law

$$
m U_{1,2}=-\frac{1}{12 \operatorname{Pr}} \frac{\partial p_{1,2}}{\partial x} .
$$

It is useful to note that the pressure distribution is not required within the narrow channel approximation under isobaric conditions. The pressure profiles can be calculated a posteriori from Eq. (28). 
The boundary conditions appropriate for Eqs. (22)-(27) are easily derived from Eqs. (14)(15):

$$
\begin{array}{ll}
x \rightarrow-\infty: & \theta_{1}=Y_{1}-1=U_{1}-1=\partial \theta_{2} / \partial x=\partial Y_{2} / \partial x=0, \\
x \rightarrow+\infty: & \theta_{2}=Y_{2}-1=U_{2}+1=\partial \theta_{1} / \partial x=\partial Y_{1} / \partial x=0 .
\end{array}
$$

From Eq. (25) and (29) it follows that $\rho_{1} U_{1}=1$ and $\rho_{2} U_{2}=-1$ for the steady solutions. Thus, as showed in [26] the computational domain can be reduced to $-\ell / 2<x<\ell / 2$ imposing

$$
\begin{array}{ll}
x=-\ell / 2: & m \theta_{1}-d \theta_{1} / d x=m\left(Y_{1}-1\right)-L e^{-1} d Y_{1} / d x=d \theta_{2} / d x=d Y_{2} / d x=0 \\
x=\ell / 2: & m \theta_{2}+d \theta_{2} / d x=m\left(Y_{2}-1\right)+L e^{-1} d Y_{2} / d x=d \theta_{1} / d x=d Y_{1} / d x=0 .
\end{array}
$$

This type of boundary conditions was used in [13]. Evidently, such a domain reduction can be applied exclusively within the narrow-channel approximation and it fails in unsteady or twodimensional cases.

\section{Numerical results}

The main aim of the present study is to compare the solutions based on the two-dimensional formulation given by Eqs. (3)-(9) and calculated for finite values of $a=H / \delta_{T}$ with those obtained from Eqs. (22)-(26) derived as $a \rightarrow 0$. It should be noted that steady-state solutions exist only for $m \geq 1$ in the given burner configuration. For $m<1$ the flame can enter and propagate inside the adiabatic segments.

In accordance with the results obtained previously in various studies, for example see [1113], two steady solutions exist for values of $m$ in the interval $1<m<m_{c}$ and no steadystates exist for $m>m_{c}$. Consider the rightward-flow channel. The first branch correspond to solutions with $x_{f 1}<0$ : the flame is situated in the first half of the heat-exchanging segment (thus, $x_{f 2}>0$ ). When the flow rate $m$ increases but remains below $m_{c}$, the flame moves towards the center of this segment. A second solution for the same value of $m$ exists and corresponds to a flame located in the second half of the heat-exchanging segment, $x_{f 1}>0$. As previously shown in [12] the first-branch solutions, with $x_{f 1}<0$, are stable and the second branch solutions are unstable.

The question of the stability of steady-state solutions can also be analyzed by the following reasoning, although this is not a rigorous mathematical analysis of the problem. In fact, when the flame in the first channel (gas flow to the right) is in the left half of the heat exchange segment (and in the second channel it is in the right half, correspondingly), its random shift to the right 
leads to an increase in the length of the heat exchange zone whose length is $\ell / 2-\left|x_{f 1}\right|$. This leads to an increase in flame temperature and, as a consequence, to an increase in flame velocity with respect to gas: the flame moves to the left. Conversely, a random shift to the left leads to the opposite effect. As a result, the position of the flame is stable with respect to the wall.

A directly opposite situation is observed when the flame in the first channel is in the right half of the heat exchange segment. It is easy to see that if the flame accidentally shifts to the right, the length of the heat exchange zone decreases, resulting in a decrease in the flame velocity relative to the gas. Thus, the flame shifts to the right even more, which means that its position is unstable. The present study deals with all the steady solutions only and the rigorous analysis related with their stability will be reported elsewhere.

Anticipating the numerical results, the maximum temperature achieved along the first branch is slightly higher than that obtained along the second branch for the same value of $m$. For this reason, in the following, we will denote the first and the second branches as the upper and lower branches referring to the maximum temperature values. This classification may fail near $m \approx m_{c}$ in sufficiently wide channels where the flame is strongly curved. For $m \approx m_{c}$ the definition of $x_{f}$ as a point laying at the channel midplane (where the reaction rate $\omega$ takes its maximum value) does not reflect completely the flame position.

\subsection{Two-dimensional results}

Figure 2 shows the typical steady-state response curves calculated from Eqs. (3)-(9) with $a=1$, $h_{w}=0.5, K=0.1$ and $\ell=30$. The left plot shows the maximum temperature reached in the channel, $\theta_{\max }$, which in this article is identified as the flame temperature, $\theta_{f}$, and the right plot gives the flame position. The solid and dashed lines represent the upper and lower branches, respectively. One can see that with increasing values of $m$ the maximum temperature grows whereas the flame shifts to the center of the heat conducting segment. There is no solution for flow rates above a certain critical value. Calculations along the lower (unsteady) branch were not carried out for the lowest values of $m$ due to numerical stiffness.

Figure 3 illustrates the steady states corresponding to the upper and lower branches for $m=$ 3.95 marked with open circles in Fig. 2. For the upper-branch (stable) solution $\left(x_{f 1}<0\right)$, the mixture is heated gradually after entering into the heat exchange section (beginning from $x=-15$ ) by heat coming from the adjacent channel. Thereafter, a rapid temperature increase takes place followed by the flame. A zone of constant temperature is situated behind the flame where the temperature is equal to the maximum temperature. There is no heat exchange within 

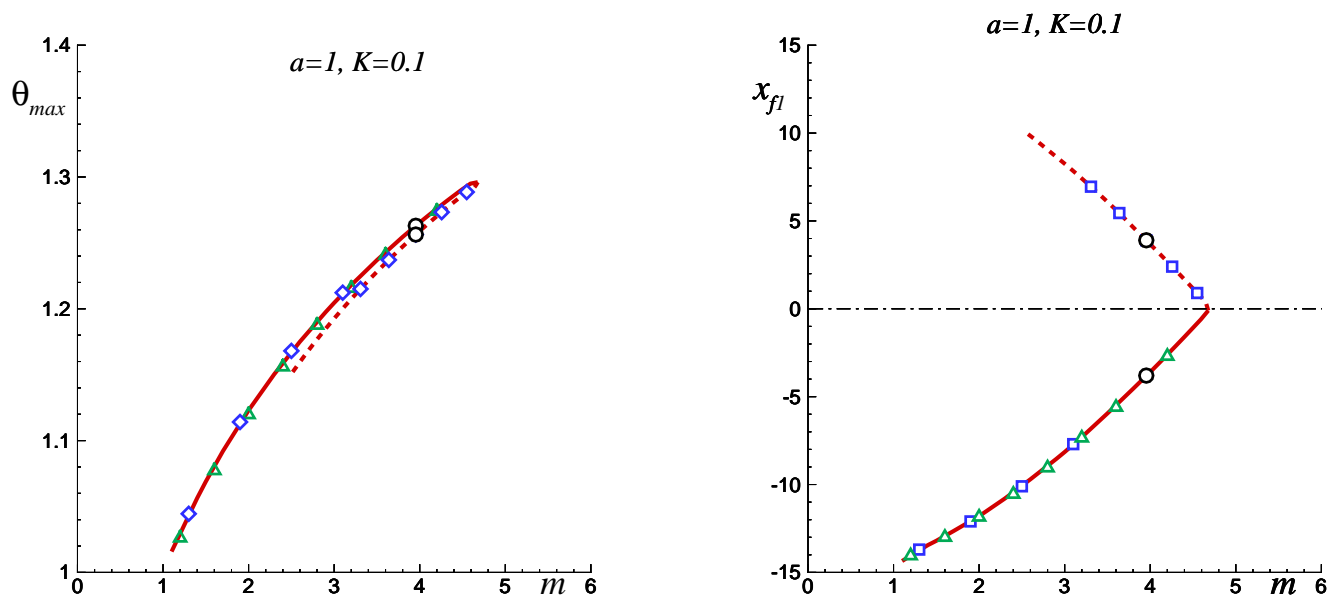

Figure 2: The maximum temperature $\theta_{\max }$ (left plot) and the flame position $x_{f 1}$ (right plot) in the rightward flowing channel versus the flow rate $m$, computed for $a=1, K=0.1, \ell=30$, $\beta=10, q=5, L e=1$ and different $h_{w}$. The solid and dashed lines represent the first and second branches, respectively, calculated for $h_{w}=0.5$; the diamond and delta symbols show the cases with $h_{w}=0.2$ and $h_{w}=1$, respectively. The open circles indicate the states plotted in Fig. 3.

this zone because the temperature is identical in the rightward and leftward flowing channels. Finally, a region with decreasing temperature appears where the burnt mixture transfers heat to the adjacent channel with fresh cold mixture flowing in the opposite direction.

The temperature distributions obtained for the lower-branch (unstable) solution are notably different. One can see that for this solution a constant temperature zone precedes the flame while a region with decreasing temperature begins just after the flame. The presence of the zone with decreasing temperature behind the flame produces a slightly lower flame temperature in comparison with the upper branch solution. This explains why, for the same value of $m$, the flame temperature along the second branch plotted with a dashed segment in Fig. 2 (left) is lower than that along the upper branch (a solid line).

One can see that for both branches the final temperature at the outlet of the heat-exchange section is exactly equal to the adiabatic flame temperature $\theta=1$. This should not be surprising, because heat losses to the exterior are neglected in the present calculations.

It is notable that the temperature distributions in the channels and the flame positions are 

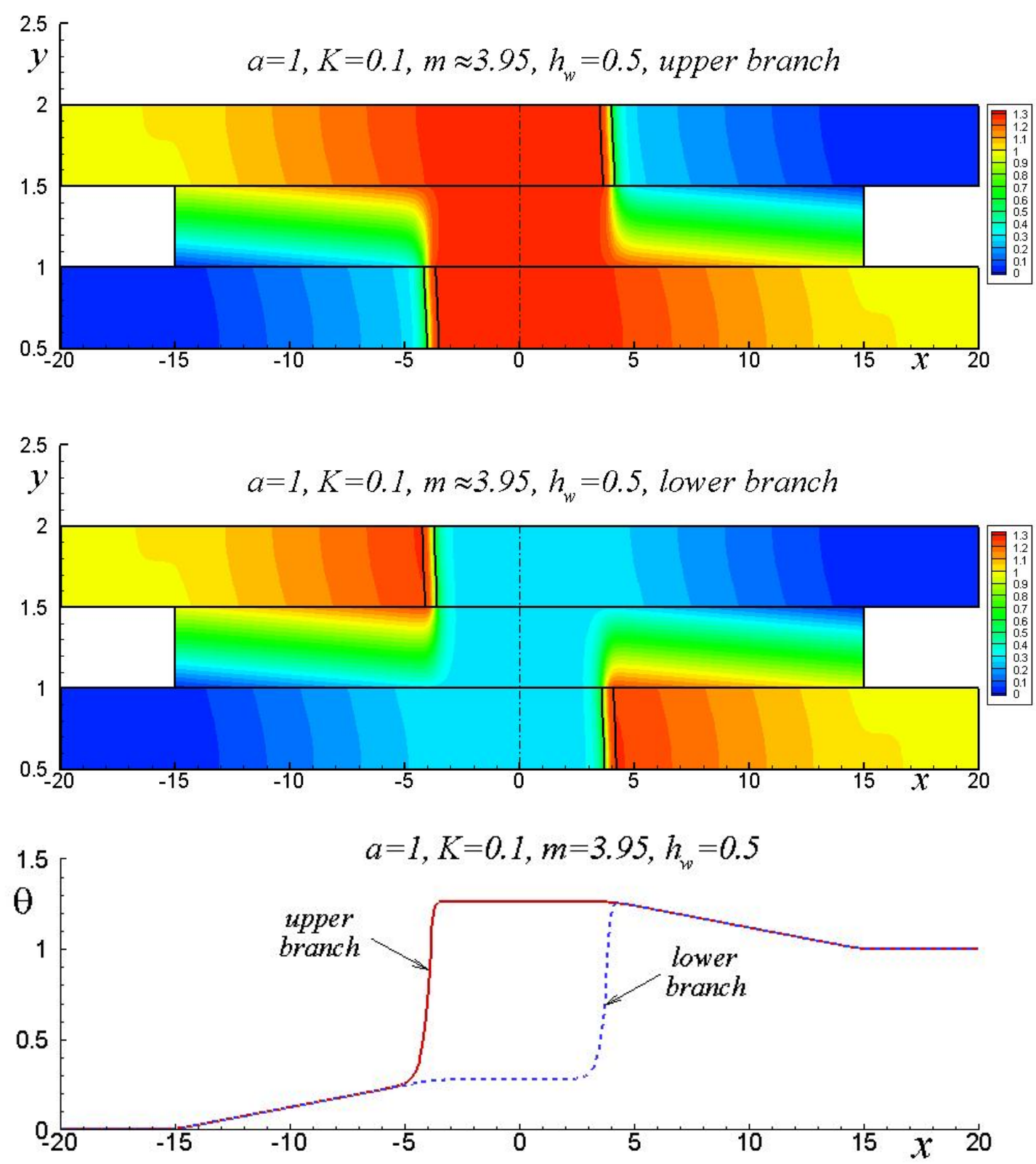

Figure 3: Temperature (color) and reaction rate (thick black lines) contour plots illustrating the states from the upper (upper plot) and lower (middle plot) branches; temperature profiles along the channel midplane in the rightward-flow channel (lower plot), for $a=1, K=0.1, m=3.95$, $\ell=30, h_{w}=0.5, \beta=10, q=5$; the corresponding states are marked with open circles in Fig. 2. 

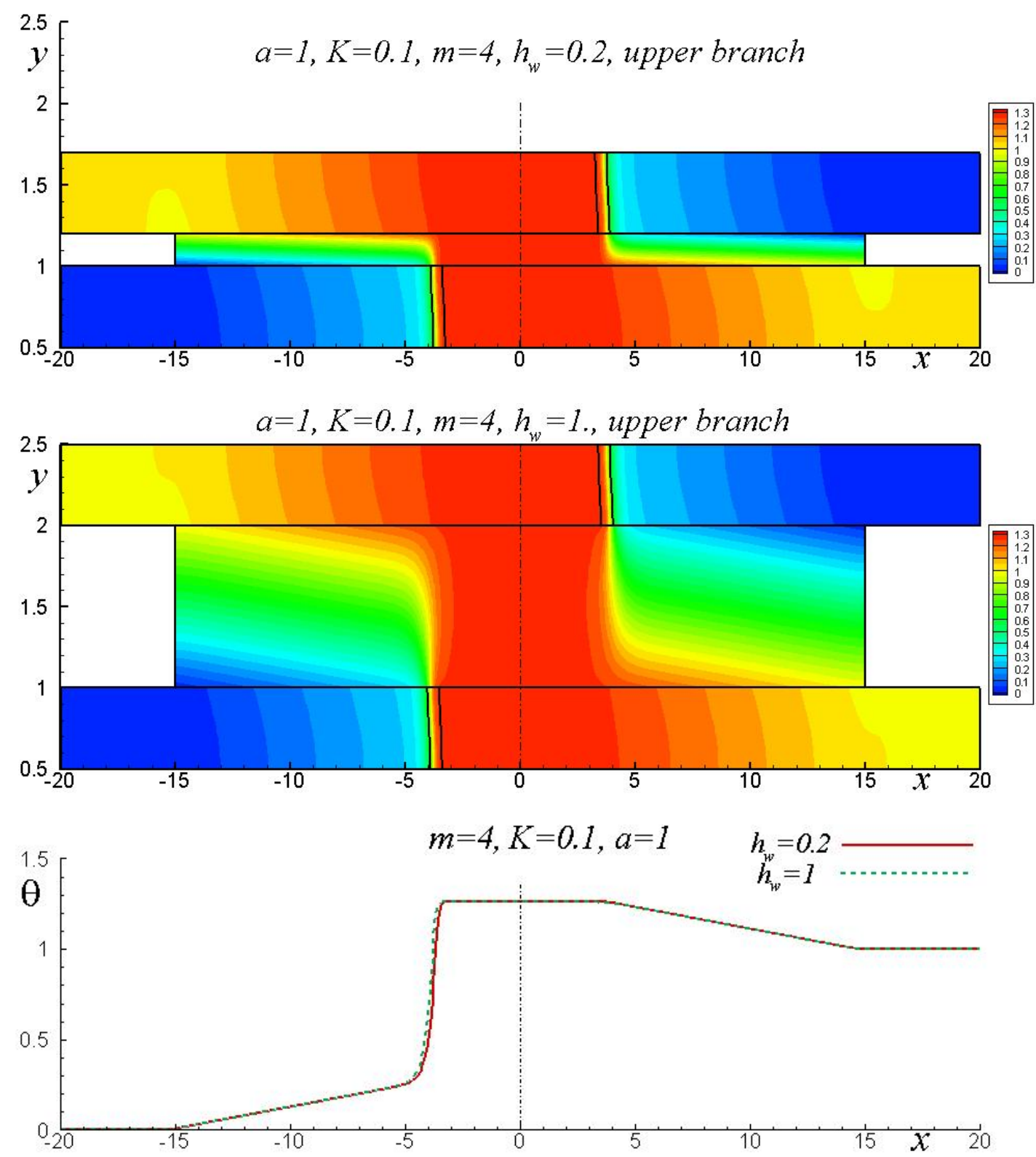

Figure 4: Two upper plots: temperature (color) and reaction rate (thick black lines) contour plots illustrating the flame from the upper branch calculated with $h_{w}=1$ and 0.2 ; all calculations for $a=1, m=4, K=0.1, \ell=30, \beta=10, q=5$; lower plot: the axial temperature profiles in the rightward flow channel.

practically independent of the wall thickness $h_{w}$, at least when $h_{w}$ remains of order unity. This is illustrated in Fig. 2 where the cases $h_{w}=1$ and 0.2 are plotted with diamond and delta 
symbols. Apparently, this occurs by virtue of parameter $K$ defined by Eq. (16). Figure 4 shows the temperature contour plots, the flame positions and the axial temperature profiles along the rightward flow channel calculated with $m=4, K=0.1$ and two different $h_{w}$.

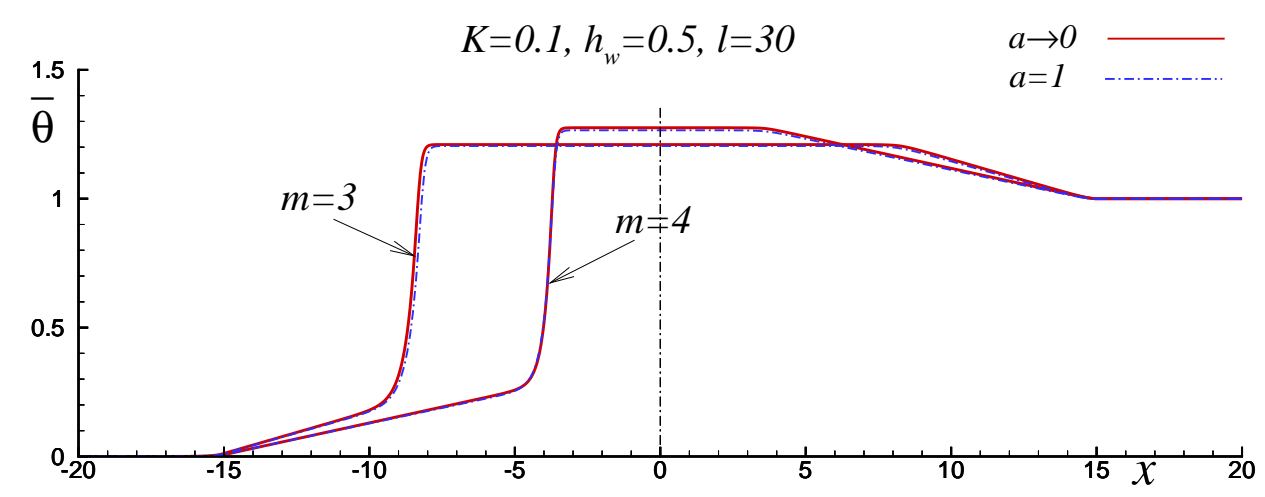

Figure 5: Comparison of the average temperature distributions calculated with $a=1$ (dashed lines) and those based on the narrow channel approximation (solid lines) for $m=3,4$ and $K=0.1, \ell=30, h_{w}=0.5, \beta=10$ and $q=5$.

\subsection{Comparison of $2 \mathrm{D}$ and $1 \mathrm{D}$ flames}

The principal goal of the present study is to evaluate the accuracy of the narrow-channel approximation developed in Section 3. With this aim in view we introduce the temperature averaged across the channel height,

$$
\bar{\theta}(x)=\int_{0}^{1} \theta(x, y) d y .
$$

Figure 5 shows the temperature distributions obtained within the narrow-channel approximation (solid lines) and the average temperatures (dashed lines) calculated for $a=1, K=0.1$ and $h_{w}=0.5$ for the flow rates $m=3$ and 4 . One can see a good agreement in the results.

Similar results were obtained for cases with shorter heat exchange segments. Figure 6 shows the temperature profiles calculated in the $a \rightarrow 0$ limit and the averaged temperatures for several values of $a$ and $K=0.05$. One can see that the one-dimensional model (the narrow-channel approximation) works reasonably well up to $a=2$. Apparently, the differences in temperature distributions appearing for higher values of $a$ occur due to the flame curvature in wider channels.

Comparison of the numerical results obtained in the framework of the full two-dimensional model and the asymptotic one-dimensional model show that the differences between them rapidly 


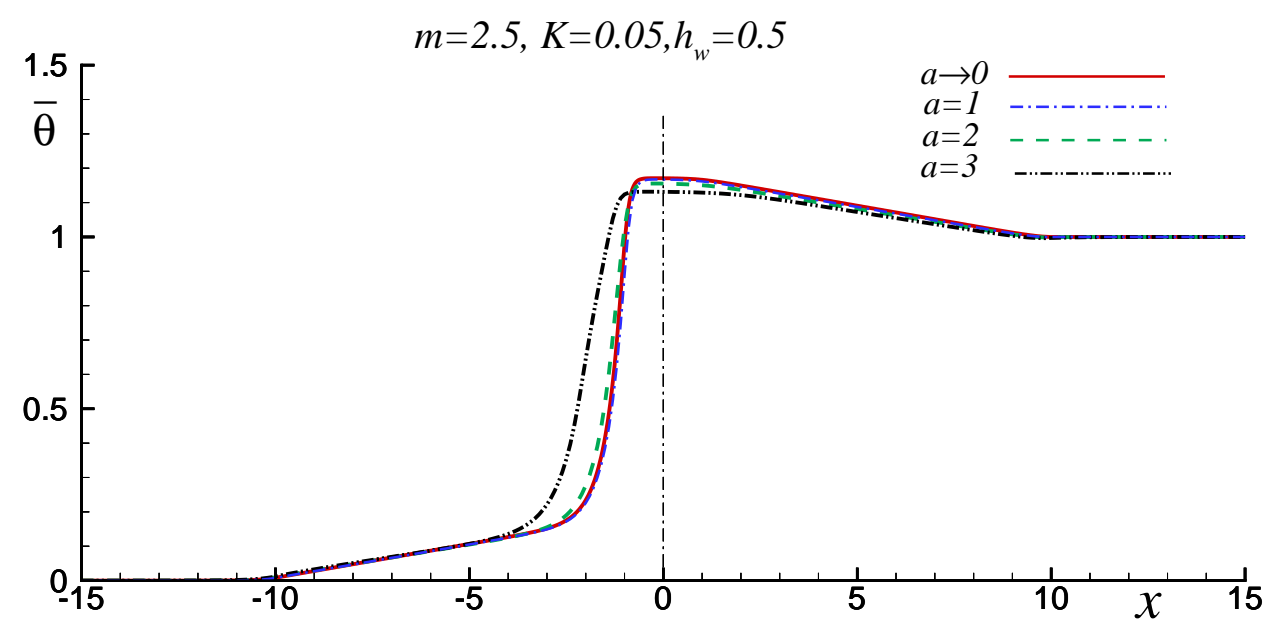

Figure 6: Comparison of the temperature profiles based on the narrow-channel approximation (solid line) and the average temperatures calculated for $m=2.5, K=0.05, \ell=20, \beta=10$, $q=5$ and various $a$.

increase for $a$ large, as expected. Figure 7 (upper plot) shows the temperature distribution (color), reaction rate (thick black lines) and stream function isolines (white lines) for $a=5$. The lower plot of Figure 7 compares the distribution of the average temperature (solid line) and the temperature obtained from the one-dimensional model (dashed line). It can be seen that the transverse distribution of the reaction rate deviates significantly from the flat profile observed at small a. As a consequence of this, the temperature averaged over the channel cross section and the temperature obtained from the one-dimensional calculation differ noticeably. We also show the isolines of the stream function in this figure. The point is that for the narrower channels shown in the previous figures the streamlines are practically straight lines. In Fig. 7 one can see the deviation of the flow field due to the action of the curved flame through the thermal expansion effect.

It is interesting, that the numerical simulations carried out for the branch with $x_{f 1}>0$ (lower branch) in the frame of the narrow channel approximation reveal the existence of non-symmetric solutions. These are apparently unstable, and therefore of minor importance. Nevertheless we plot in Fig. 8 an example of the temperature profiles calculated for $m \approx 3.338, K=0.1$ and $\ell=50$. One can see that distributions in the rightward flow and leftward flow channels are not symmetric with respect to $x=0$. The outlet temperatures are also different. Nevertheless, it should be noted that the law of conservation of energy is of course verified. Positive deviation 

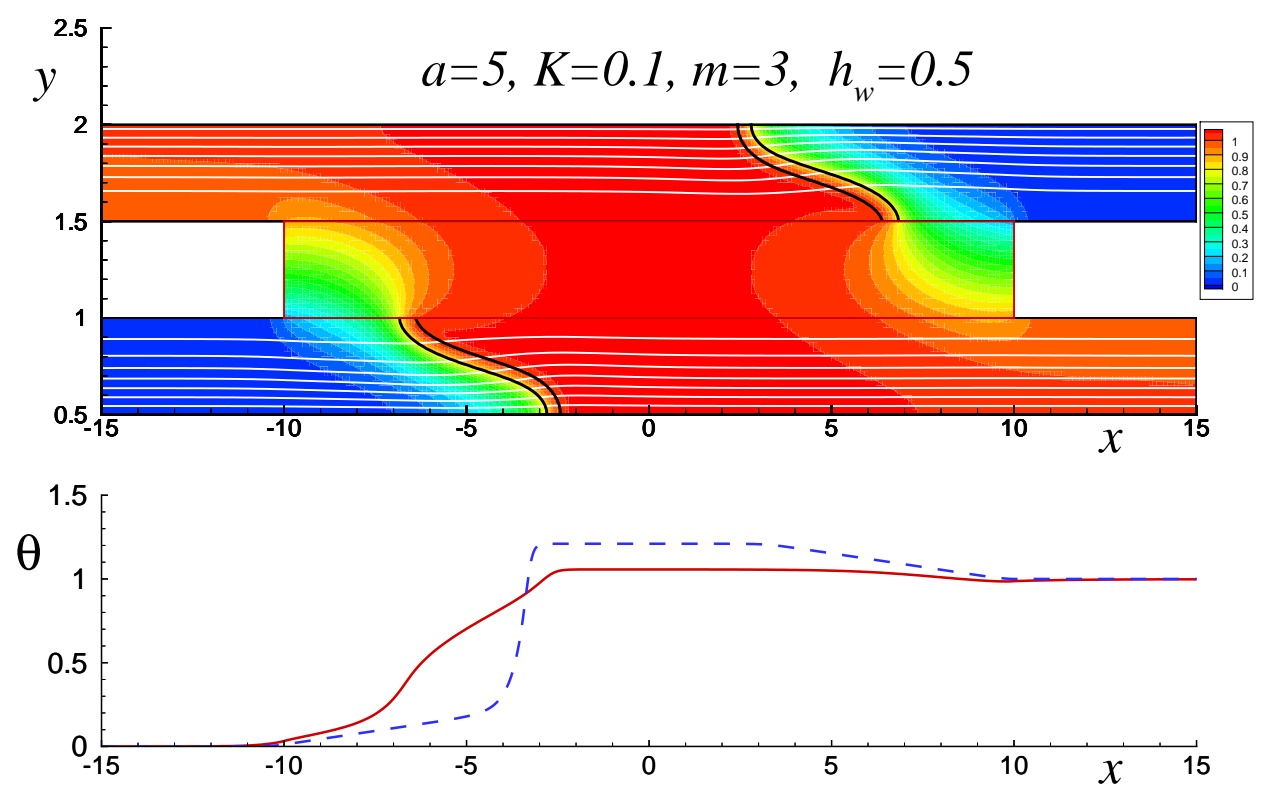

Figure 7: Upper plot: temperature (color), reaction rate (thick black lines) and stream-lines (white lines) contour plots illustrating the flame from the upper branch calculated with $a=5$, $m=3, K=0.1, h_{w}=0.5, \ell=20, \beta=10, q=5$; lower plot: the averaged temperature profile (solid line) and 1D temperature (dashed line) in the rightward flow channel.

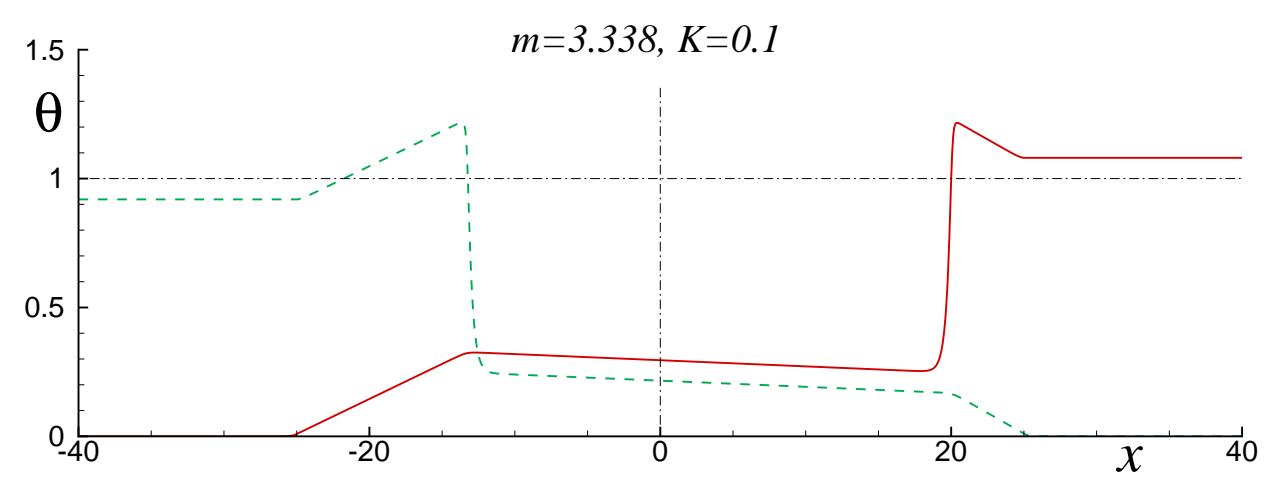

Figure 8: An example of non-symmetric solutions obtained in the 1D model: the temperature profiles in the rightward flow (solid line) and leftward flow (dashed line) channels calculated for $m \approx 3.338, \ell=50, K=0.1, \beta=10$ and $q=5$.

of temperature from adiabatic in one channel is strictly compensated by the opposite deviation in the other. 


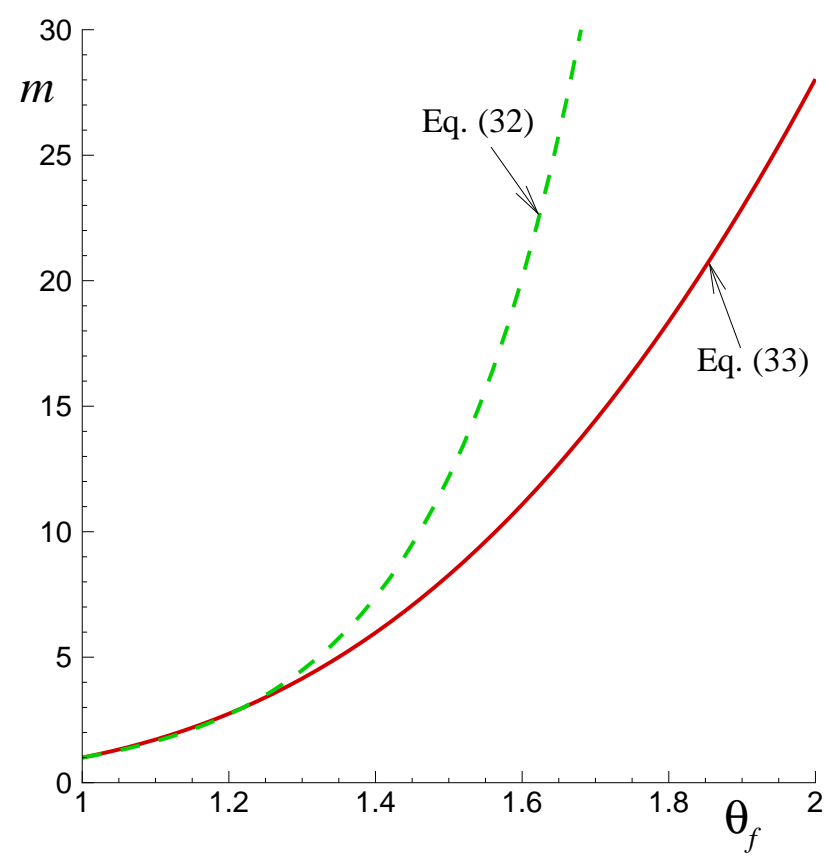

Figure 9: Profiles given by Eq. (33) (a solid line) and Eq. (32) (a dashed line) plotted for $\beta=10$ and $q=5$.

\section{Large activation energy limit}

It is interesting to compare the numerical results calculated with the spatially distributed reaction rate (finite $\beta$ ) and those of large activation energy approximation $(\beta \rightarrow \infty)$ known as the flamesheet model. In point of fact, the mathematical formulation of the flame sheet model consists in substituting the reaction rate given by Eq. (10) with $\omega=m \delta\left(x-x_{f}\right)$, where $\delta(\cdot)$ is the Dirac delta function. This approximation was used in [11-13] for flames in heat-recirculating devices.

For the steady-state solutions, the flame velocity with respect to the unburned mixture should be equal to the flow velocity at the flame region. The relation between the flow rate $m$ and the flame temperature $\theta_{f}$ used in [11-13] was in the form

$$
m=e^{\beta\left(\theta_{f}-1\right) / 2} .
$$

In the recent study [27] the propagation of planar isobaric flames in narrow channels with heat losses was analyzed asymptotically using the method of matched asymptotic expansions 
for $\beta \gg 1$. Within this method, the thickness of the reaction zone is of order $\beta^{-1}$ (a thin flame sheet), outside which the reaction rate remains exponentially small. In particular, the direct relation between the flame velocity and the flame temperature was obtained as follows

$$
m=\mu(G)^{-1 / 2} \frac{1+q \theta_{f}}{1+q} \cdot \exp \left\{\frac{\beta}{2} \frac{\left(\theta_{f}-1\right)}{\left(1+q \theta_{f}\right) /(1+q)}\right\} .
$$

where $G=-d \theta /\left.d x\right|_{x=x_{f}-}$ denotes the temperature gradient just behind the flame sheet (at the burnt side). Here the more general expression obtained in [27] has been adopted for the $\rho^{2}$-dependence of the reaction rate $\omega$ given by Eq. (10) and constant transport properties. This expression obtained asymptotically in a rigorous manner is quite different from its simplified counterpart given in Eq. (32). Even if these two expressions are close near $\theta_{f} \approx 1$, a significant difference is observed with increasing values of $\theta_{f}$, as illustrated in Fig. 9 where $\mu=1$ was used.

Following [27], the value of $\mu$ in Eq. (33) is determined by considering the inner flame region. Finally, $\mu$ depends on the temperature gradient just behind the flame and can be approximated as $\mu \approx 1-\mu_{1} G$, where $\mu_{1}=1.344046$ was calculated numerically. It is important to note that in the heat recirculating devices considered in the present study the temperature behind the flame remains constant for the upper-branch (stable) solutions thus being equal to $\theta_{f}$. This can be seen in Fig. 5, for example, where the curves corresponding to the $a \rightarrow 0$ limit are shown with solid lines. Thus, $G=0$ and $\mu=1$ should be used in Eq. (33) for the upper-branch solutions. Only this branch is considered in the present section.

Relation (33) states that for $\beta \gg 1$ the flame temperature, $\theta_{f}$, depends on the flow rate $m$ (and the kinetic parameters $\beta$ and $q$ ) solely, remaining independent on the heat exchange parameter $K$. Finally, the flame temperature takes the form

$$
\theta_{f}=\frac{\beta(1+q)^{2}-2 q Z}{2 Z q^{2}}, \quad \text { with } \quad Z=W\left(\frac{\beta(1+q) e^{\beta(1+q) / 2 q}}{2 q m}\right),
$$

where $W(\xi)$ is the Lambert function defined by the equation $W(\xi) e^{W(\xi)}=\xi$. It is a multivalued function with an infinite number of branches such that for each non-zero value of $\xi$ there is an infinite number values of $W$, most of them complex. Here the principal branch is used.

Equation (34) provides the flame temperature, $\theta_{f}$, as a function of $m$ for $\beta \gg 1$. Let us assume that the temperature profiles in adjacent channels are symmetric with respect to $x=$ 0 , namely $\theta_{1}(x)=\theta_{2}(-x)$ and $x_{f 2}=-x_{f 1}$. Hence calculations of the flame position $x_{f 1}$, 
unknown beforehand, are reduced to

$$
\left\{\begin{aligned}
m d \theta_{1} / d x & =d^{2} \theta_{1} / d x^{2}-K\left(\theta_{1}-\theta_{2}\right), \\
-m d \theta_{2} / d x & =d^{2} \theta_{2} / d x^{2}+K\left(\theta_{1}-\theta_{2}\right),
\end{aligned}\right.
$$

to be solved for $-\ell / 2<x<x_{f 1}$ subject to the following boundary conditions

$$
\begin{array}{ll}
x=-\ell / 2: & m \theta_{1}-d \theta_{1} / d x=d \theta_{2} / d x=0, \\
x=x_{f 1}: & \theta_{1}=\theta_{2}=\theta_{f}, \quad d \theta_{1} / d x=m\left(\theta_{f}\right) .
\end{array}
$$

Here $x_{f 1}<0$ for the upper branch and $m=m\left(\theta_{f}\right)$ is given by Eq. (33) with $\mu=1$. Notice, that the third condition imposed at $x=x_{f 1}$ allows to calculate $x_{f 1}$.

Equations (35) are linear with respect to $\theta_{1}$ and $\theta_{2}$ allowing an analytical treatment. This was carried out by using MAPLE, which facilitates much the straightforward algebraic calculations. Even though the general expressions for $\theta_{1}$ and $\theta_{2}$ are relatively simple, the final equation to determine the flame position $x_{f 1}$ results to be very long and writing it down does not reveal much about the nature of the solution. The parametric study presented below is based on the numerical evaluation of this analytical expression.

Figure 10 shows the flame position in the rightward-flow channel, $x_{f 1}$, as a function of the flow rate $m$ for different values of $\beta$. The solid lines were calculated using the spatially distributed reaction rate stated in Eq. (10). The dashed curves were obtained from Eqs. (35)(36) using Eq. (33). One can see that with increasing values of $\beta$ the asymptotic dashed-line curves based on the $\delta$-function approximation for the reaction rate approach remarkably well the numerical solid-line curves calculated using the spatially distributed reaction rate. This figure validates also the asymptotic relation given by Eq. (33) for superadiabatic flames.

Stating $x_{f 1}=0$ in Eqs. (35)-(36) allows to calculate the critical value $m_{c}$ above which, for $m>m_{c}$, there are no solutions. This property is plotted in Fig. 11 where the curves calculated using Eq. (32) and (33) are shown with dashed and solid lines, respectively.

\section{Concluding discussion}

Building simplified models for numerical analysis is sometimes difficult, especially when a significant number of physical processes must be included. One way to do this is to use asymptotic methods. However, after a simplified model is built, a legitimate question arises about the extent of its application. As a rule, the answer to this question cannot be obtained from the asymptotic 


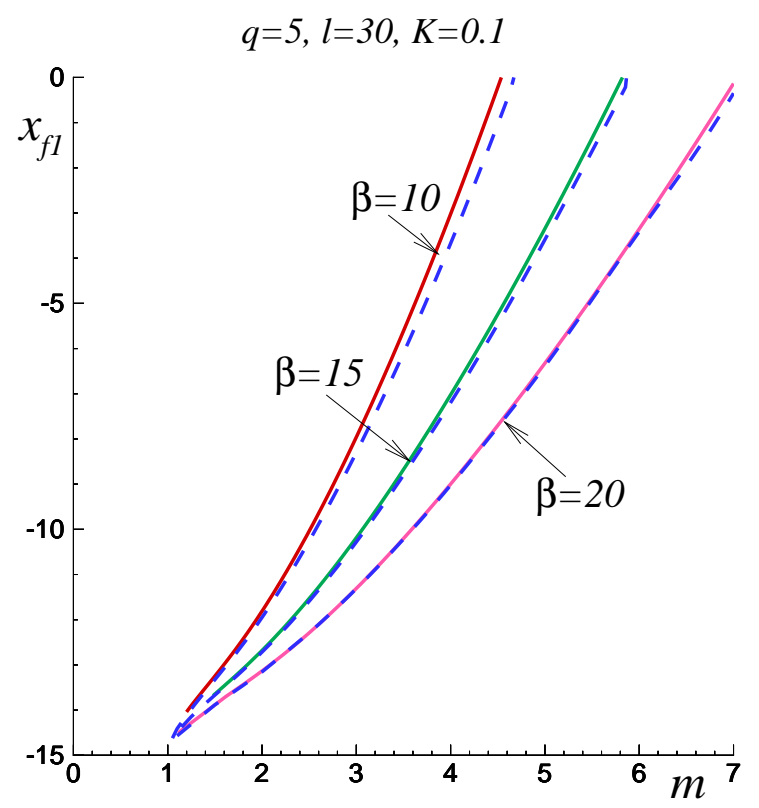

Figure 10: Comparison of the flame position $x_{f 1}$ calculated with spatially distributed reaction rate (solid lines) and with the flame-sheet model approximation with Eq. (33) used for $m\left(\theta_{f}\right)$ for various $\beta$; all curves are plotted for $q=5, \ell=30$ and $K=0.1$.

construction itself, and comparison with the results obtained in the framework of a more general model is extremely necessary.

This is precisely the situation that arises when studying processes in small-scale combustion devices with heat recirculation, where heat transfer and heat exchange play a decisive role. In addition to the parameters that characterize the combustible mixture and the transport processes in gas and solid phases, the geometric characteristics of the device should be included in the model. The total number of parameters can be significant, thereby making it difficult to study the parametric optimization of its operation.

A widely used class of models applying recently for such devices is the one-dimensional models. In fact, it is assumed that although the profile of the mixture flow velocity in a channel has a Poiseuille shape, the effective narrowness of channels reduces the transverse distributions of temperature and concentrations of species to the effective average values calculated across the channel cross-section. On the other hand, the strong sensitivity of the rate of the combustion chemical reaction, especially on temperature, can cast doubt on the results obtained by averaging 


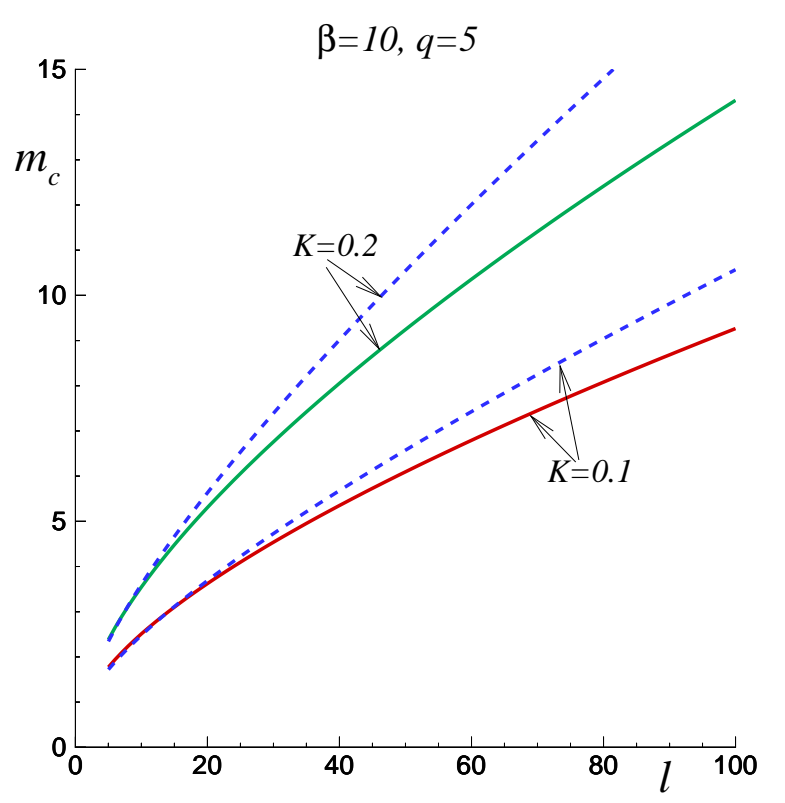

Figure 11: The maximum flow rate $m_{c}$ (corresponding to $x_{f 1}=0$ ) as function of $\ell$ plotted using Eq. (33) (solid lines) and Eq. (32) (dashed lines) for $K=0.1$ and 0.2, all curves with $\beta=10$ and $q=5$.

the transverse distributions. The correct accounting of lateral heat transfer to nearby channels through the side walls can also cause difficulties.

In the present work, the one-dimensional model is obtained strictly asymptotically by expansion in power series of a small parameter. This parameter is the ratio of the thermal width of the flame to the thickness of the channel, when the thickness of the separating walls between the channels is in the same order as the thickness of the channels. Despite the fact that, for simplicity of the formulation of the problem, heat losses to the environment were not taken into account in the present study, it is obvious that they can also be taken into account asymptotically within the same approximation. This would lead to the appearance of a heat sink additional term in the one-dimensional equation for energy balance.

By comparing with the results obtained in the framework of the full two-dimensional model, it was demonstrated that the application limits of the one-dimensional model is much wider than it would be, based on the smallness of the expansion parameter. Moreover, it is shown that the one-dimensional model gives quantitatively correct results even when a formally small 
parameter is taken of order unity or even larger.

\section{Acknowledgment}

The authors acknowledge the support of Spanish MEC under project \#ENE2015-65852-C2-2-R (MINECO/FEDER, EU).

\section{References}

[1] A.C. Fernández-Pello, Micropower generation using combustion: issues and approaches, Proc. Combust. Inst. 29 (2002) 883-899.

[2] D. Dunn-Rankin, E.M. Leal , D.C. Walther, Personal power systems, Prog. Energy Combust. Sci. 31 (2005) 422-465.

[3] Y. Ju, K. Maruta, Microscale combustion: technology development and fundamental research, Prog. Energy Combust. Sci. 37 (2011) 669-715 .

[4] D.C. Walther, J. Ahn, Advances and challenges in the development of power-generation systems at small scales, Prog. Energy Combust. Sci. 37 (2011) 583-610 .

[5] K. Maruta, Micro and mesoscale combustion, Proc. Combust. Inst. 33 (2011) 125-150.

[6] N.S. Kaisare, D.G. Vlachos, A review on microcombustion: fundamentals, devices and applications, Prog. Energy Combust. Sci. 38 (2012) 321-359.

[7] S.A. Lloyd, F.J. Weinberg, A burner for mixtures of very low heat content, Nature 251 (1974) 47-49.

[8] S.A. Lloyd, F.J. Weinberg, Limits to energy release and utilisation from chemical fuels, Nature 257 (1975) 367-370.

[9] A.R. Jones, S.A. Lloyd, F.J. Weinberg, Combustion in heat exchangers, Proc. R. Soc. Lond. A. 360 (1978) 97-115.

[10] P. Ronney, Analysis of non-adiabatic heat-recirculating combustors, Combust. Flame 135 (2003) 421-439. 
[11] Y. Ju, C.W. Choi, An analysis of sub-limit flame dynamics using opposite propagating flames in mesoscale channels, Combust. Flame 133 (2003) 483-493.

[12] R.V. Fursenko, S. S. Minaev, Flame stability in a system with counterflow heat exchange, Combust. Explos. Shock Waves 41(2) (2005) 133-139.

[13] I. Schoegl, J.L. Ellzey, Superadiabatic combustion in conducting tubes and heat exchangers of finite length, Combust. Flame 151 (2007) 142-159.

[14] J.A. Federici, D.G. Vlachos, A computational fluid dynamics study of propane/air microflame stability in a heat recirculation reactor, Combust. Flame 153 (2008) 258-269.

[15] I.M. Schoegl, J.L. Ellzey, Numerical investigation of ultra-rich combustion in counter ow heat exchangers, Combust. Sci. Technol. 182 (2010) 1413-1428.

[16] V.N. Kurdyumov, M. Matalon, Analysis of an idealized heat-recirculating microcombustor, Proc. Combust. Inst., 33(2) (2011) 3275-3284.

[17] M. Sánchez-Sanz, Premixed flame extinction in narrow channels with and without heat recirculation, Combust. Flame 159 (2012) 3158-3167.

[18] E. Fernández-Tarrazo, M. Sánchez-Sanz, R. Fursenko, S. Minaev, Multiple combustion regimes and performance of counter-flow microcombustor with power extraction, Math. Modell. Nat. Phen. 13 (2018) UNSP 52.

[19] D. Fernández-Galisteo, E. Fernández-Tarrazo, C. Jiménez, V.N. Kurdyumov, Analysis of an idealized counter-current microchannel-based reactor to produce hydrogen-rich syngas from methanol, Int. J. Hydr. Eng. 44 (2019) 23807-23820.

[20] V.N. Kurdyumov, Lewis number effect on the propagation of premixed flames in narrow adiabatic channels: Symmetric and non-symmetric flames and their linear stability analysis, Combust. Flame 158 (2011) 1307-1317.

[21] V.N. Kurdyumov, C. Jiménez, Propagation of symmetric and non-symmetric premixed flames in narrow channels: Influence of conductive heat-losses, Combust. Flame 161 (2014) 927-936. 
[22] D. Fernández-Galisteo, C. Jiménez, M. Sánchez-Sanz, V.N. Kurdyumov, Effects of stoichiometry on premixed flames propagating in narrow channels: symmetry-breaking bifurcations, Combust. Theory Modell. 21 (2017) 1050-1065.

[23] A. Dejoan, C. Jiménez, V.N. Kurdyumov, Critical conditions for non-symmetric flame propagation in narrow channels: Influence of the flow rate, the thermal expansion, the Lewis number and heat-losses, Combust. Flame 209 (2019) 430-440.

[24] W.B. Bush , F.E. Fendell, Asymptotic analysis of laminar flame propagation for general Lewis number, Combust. Sci. Technol. 1 (1970) 421-428 .

[25] V.N. Kurdyumov, M.Matalon, Flame acceleration in long narrow open channels, Proc. Comb, Ints. 34 (2013) 865-872.

[26] P.V. Dankwerst, Continuous flow system: distribution of residence times, Chem. Eng. Sci. 2 (1953) 1-13.

[27] V.N. Kurdyumov, Propagation of premixed isobaric flames in narrow channels with heatlosses: The asymptotic analysis revised and reliance on the flame-sheet model, Combust. Flame. 206 (2019) 138-149. 